\title{
Optimising Blue Fluorescent Protein (BFP) for use as a mammalian reporter gene in parallel with Green Fluorescent Protein (GFP).
}

\author{
Etienne Joly \\ Running title: "Blue and green should never be seen..." \\ This work was carried out whilst I worked at The Babraham Institute, \\ Cambridge, CB2 4AT, UK \\ My current address is: IPBS, UMR CNRS 5089 \\ 205 route de Narbonne, 31077 Toulouse Cedex \\ atn@cict.fr
}

Note : This manuscript was written in October 1997, and was, at the time, successively rejected from the Journal of Biological Chemistry and from

Nucleic Acids Research (the reason given both times being that the observations reported were not sufficiently interesting for these journals). Because of more pressing personal priorities, this manuscript has been sitting in my drawers since January 1998. Readers should therefore not be surprised that some appropriate more recent references will be missing. The publication of a report describing an optimised form of BFP in last

December's issue of Nature Biotechnology (Mena et al., Nature Biotechnology 2006, 24: 1569-71) suggested to me that some of the observations contained in this manuscript may still be of interest to some of my colleagues, and I have therefore decided to diffuse it in the form of a Nature Preceding. 
Summary:

To obtain an optimised form of BFP for use as a reporter gene in mammalian cells, the brightest available GFP form, EGFP, was mutated at 5 different positions, yielding 8 different mutagenised forms of BFP. The intensity of the fluorescent signals attained in mammalian cells with all these various versions of BFP was analysed by flow cytometry of transiently transfected COS 7 cells. The best mutant obtained can be detected readily both by flow cytometry and fluorescence microscopy, even when expressed together with GFP. To explore whether cellular localization could enhance the fluorescence signals any further, plasmid constructs were made to target optimised versions of GFP and BFP to the nucleus, the endoplasmic reticulum (ER) and the cell surface. Expression in the nucleus or ER increased the fluorescence signal by ca. $50 \%$, whereas cell surface expression resulted in a five-fold decrease compared to the ER and nuclear forms. Coexpression of GFP and BFP in the same cellular compartment did not result in any significant absorption of the blue fluorescence by GFP. Thus, targeting of GFP and BFP to various cellular compartments adds even further versatility to this convenient dual reporter-gene system. 


\section{Introduction:}

Over the past few years, Aequorea victoria 's green fluorescent protein (GFP) has proven an invaluable tool to study gene expression, protein localization and sub-cellular compartment dynamics. In addition, the production of variants with altered spectral characteristics has opened the door to the detection of several signals in parallel. Of the variants reported so far, the most useful are derived from the Y66H mutation which leads to emission of blue fluorescence [1-3]. A yellow variant form of GFP, EYFP, has also been reported recently as a potential tool for dual colour analyses [4]. The overlap of the green and yellow spectra, however, make this variant unsuitable for analysis by fluorescence microscopy in parallel with GFP, and separate detection by flow cytometry is possible only with carefully controlled electronic compensation. The fluorescence signals obtained with BFP and GFP are easily separated, but two major drawbacks of BFP are that its fluorescence is much weaker than that of GFP and the UV required for excitation results in rapid bleaching in fluorescence microscopy. So far, BFP has not been very widely used in mammalian cells as a reporter gene mainly because of these drawbacks.

GFP derivatives have been expressed efficiently in many organisms by various modifications. Firstly, efficient protein expression can be affected if the nucleic acid sequence shows a bias towards codons that are rarely used by the organism, as was demonstrated by Haas et al. on expression of HIV-1 glycoprotein, Thy-1 and GFP in human cells [5]. Several fluorescence protein (FP) sequences have now been reported where the whole nucleotide sequence has been synthetically remodelled to accommodate the codon usage of mammalian cells [5-8]. Secondly, several mutations resulting in an increase of the protein's stability at $37^{\circ} \mathrm{C}$ have been reported by different groups [9-12]. 
In addition to the $\mathrm{Y} 66 \mathrm{H}$ mutation necessary for blue fluorescence, four other substitutions were strong candidates for improving expression of BFP in mammalian cells. Firstly, Y145F, was reported to double the quantum yield of BFP compared to Y66H alone [2]. Secondly, the reversal of Thr 65 to the original Ser found in the natural form of GFP; the S65T mutation in GFP results in a brighter fluorescence and in a red shift of the absorption spectrum which makes it more compatible with the widely available optical filters used with fluorescein $[11,13]$, but it was unclear how this mutation would affect the spectrum of BFP. Finally, two mutations, V163A and S175G, were reported to increase the stability of bacterially produced GFP at $37^{\circ} \mathrm{C}[10]$. Because each of these substitutions was reported independently and mainly in bacterially produced GFP, it was not certain that they would all be beneficial for a BFP synthesised in mammalian cells. These four substitutions were, therefore, introduced in a combinatorial array to test systematically their effects on BFP expressed in mammalian cells. 


\section{Experimental Procedures:}

Plasmid constructions: The coding sequence for EGFP was initially derived from the pEGFP1 vector purchased from Clontech (acc. numb. U55761). The EGFP gene, excised by BamHI/NotI was then subcloned into these same sites in pCATCH-NLS [14]. This pcDNA1/Amp-based eukaryotic expression plasmid (Invitrogen), which was kindly provided by Christopher Hovens, has been designed to tag proteins with a FLAG epitope, a heart-muscle kinase phosphorylation site and a nuclear localization signal. Frame continuity between the CATCH-NLS tag and the EGFP sequence was subsequently obtained by filling of the BamHI site with Klenow polymerase. Untagged EGFP under the control of the same CMV promoter was obtained by excising the CATCH-NLS tag with HindIII and BamHI and ligating the blunted overhangs.

For ER-localization, SBFP and SGFP were amplified by PCR using pfu DNA polymerase (Stratagene). The upstream oligonucleotide 5'GCCTGCAGAGCAAGGGCGAGGAGCTGTTC-3' introduced a Pst1 site (shown in bold), whereas the downstream oligonucleotide 5'CGTCTAGAGCTCGTCCTTGTACAGCTCGTCCATGC-3' created a Cterminal KDEL ER-retention sequence and introduced an XbaI site (in bold) immediately after the stop codon. The PCR amplified fragments were digested with PstI and $\mathrm{XbaI}$ and cloned into the same sites in pS8DXM1. In this plasmid, which was kindly provided by Sean Munro, a 170 bp HindIII/PstI fragment encodes an immunoglobulin leader peptide. The ERtagged sequence was then subcloned back into pcDNAI/Amp with the enzymes HindIII and BamHI.

For cell surface localization, the KDEL sequences in ER-SBFP and ERSGFP were replaced by the transmembrane and intracytoplasmic domain of a rat MHC class I molecule, RT1-A ${ }^{u}[15]($ acc. numb. X82106). For this, the 
BsrGI site situated just upstream of the stop codon in EGFP was used, and filled in with Klenow. After phenol extraction, the KDEL-coding sequences were excised using XbaI, and the StuI/XbaI 350 bp fragment from pCMU-A ${ }^{u}$ [15] was cloned in their place. This resulted directly in continuous reading frames between SBFP or SGFP and the MHC class I sequence.

Site-directed mutagenesis of EGFP was carried out using a modification of the protocol in the QuikChange ${ }^{\mathrm{TM}}$ kit (Stratagene), and is described elsewhere [16]. The S175G mutation was introduced with the same protocol, using the oligonucleotide: $5^{\prime}-$

GGCGAGCTGCACGCCGCCGTCCTCGATG-3'. The coding sequences for all the BFP variants listed in Table 1 were checked as devoid of unwanted mutations by automated sequencing.

Transfection of COS 7 cells was performed as described previously [17]. When many plasmids were analysed, transfections were carried out in multiwell tissue culture dishes. In such case, the low $\mathrm{CO}_{2}$ concentration required for optimal transfection efficiency was obtained by turning off the incubator's $\mathrm{CO}_{2}$ input during the 2 hour incubation with the DNA/DEAEdextran transfection mixture.

For FACS analyses, cells were harvested by trypsinization 48 or 72 hours after transfection, washed once with PBS, and fixed in PBS/1\% formaldehyde. Fixation of cells with formaldehyde does not noticeably affect the levels of fluorescence recorded from BFP or GFP, but it results in a slight increase of autofluorescence of the cells in the green channel, and in a two-fold decrease in the blue channel (data not shown). This proved very useful for the detection of weak blue fluorescent signals such as those from plasmids 3 and 28 , since the overall effect is an increase of the signal/noise ratio for blue fluorescence. Cells could be kept at $4^{\circ} \mathrm{C}$ in $\mathrm{PBS} / 1 \%$ formaldehyde for over 2 weeks without any noticeable decrease of the fluorescence intensity of GFP or BFP (not shown). FACS analyses were 
performed with CellQuest software on a Becton Dickinson FACS Vantage, using two different Spectra Physics 2020 lasers on two independent excitation lines. For GFP analyses, a tuneable laser was used on $488 \mathrm{~nm} / 400$ $\mathrm{mW}$, and emissions recorded through the standard 530/30 fluorescein filter. For BFP analyses, a 351-363 nm UV laser was used at 300mW, and emissions recorded through a 460/40 filter obtained from Ealing Optics (ref. 35-5024). $10^{5}$ cells, gated for FSC/SSC, were collected for each sample.

For observation by fluorescence microscopy, cells were trypsinized and seeded onto sterile coverslips $48 \mathrm{~h}$ after transfection, and analysed the following day. After washing once with PBS, cells were either fixed in $\mathrm{PBS} / 1 \%$ formaldehyde, or mounted directly in PBS. Pictures shown in Figures 2 and 4 were obtained with an Axiophot microscope (Zeiss) using a 63x oil immersion objective and Kodak 400 ASA slide film. For preparation of the composite pictures shown, slides were scanned and combined using Photoshop (without any electronic manipulation of the colour curves). For GFP, the fluorescein filter cube was used (450-490, FT510, LP520), whilst BFP was observed with a filter cube designed for DAPI stains (G365, FT395, LP420). This latter set of filters is, however, suboptimal for co-detection of GFP and BFP, since green light emitted by GFP is not eliminated by the 420nm LP filter.

Spectral characteristics of the various constructs were analysed on a PerkinElmer LS3 fluorimeter using transiently transfected COS cells in PBS-1\% formaldehyde. Constant wavelengths used were $380 \mathrm{~nm}$ and $460 \mathrm{~nm}$ for BFPs, and 460 and 540 for GFPs 
Results :

To obtain an optimised version of BFP to use in parallel with GFP in mammalian cells, a commercially available version of GFP called EGFP (Clontech) was chosen. This is reputedly the brightest version of GFP available for expression in mammalian cells $[7,8,18]$. The EGFP sequence has been adapted for mammalian codon usage, carries a canonical Kosak sequence [19], and an F64L substitution which increases the solubility and/or stability of GFP at $37^{\circ} \mathrm{C}[11,20]$. The starting material for site-directed mutagenesis was a derivative of EGFP called nls-EGFP, which expresses a form of GFP tagged with an SV40 nuclear localization signal under the control of the strong CMV promoter (for details of the construct, see Exp. Proc.). The SV40 nuclear localization signal was used because it gives a 50\% improvement in the level of expression of EGFP compared with the untargeted form (see below). It was therefore felt that it would improve detection of a possibly weak blue fluorescent signal and facilitate the comparison between the efficiency of the different mutated forms. The five mutations described above were introduced into the p-nls-EGFP plasmid using a novel simplification of the QuikChange ${ }^{\mathrm{TM}}$ method (Stratagene) [16].

Levels of fluorescence intensity attained were assessed by FACS analysis of COS 7 cells transiently transfected using the DEAE-dextran method [17, 21]. In addition to being rapid, cheap and simple, this transfection method presents other advantages. Firstly, it is possible to use minipreparations of plasmid DNA and still obtain efficiencies of transfection routinely superior to $40 \%$ [17]. Secondly, with several different reporter-genes constructs on plasmids carrying the SV40 origin of replication, I have observed repeatedly that the average levels of expression in individually transfected COS 7 cells is remarkably independent of the quality of the plasmid DNA used, and of the overall efficiency of transfection (not shown). This was important in this study because, even though all the plasmids for expression of the BFP 
variants were purified using identical protocols, most of them were prepared on different dates. The fact that the quality of the plasmid DNAs used did not detectably influence the levels of signals obtained allowed the screening of a large number clones after mutagenesis[16], and was also essential for the validity of the comparisons between plasmids prepared on different dates.

From the results shown in Table 1, one can see clearly that the most fluorescent BFP forms all contain S65, H66 and F145. In all three pairs of constructs that differ only at position 65 (5 vs 7; 18 vs 1; 28 vs 10), T65, which results in brighter red-shifted fluorescence for GFP, results in a signal that is 3-4 fold weaker in BFP. Similarly, in the pairs that differ only at position 145 ( 3 vs 5; $10 v s$ 1; 28 vs 18), F145 fluorescence increased 2-fold compared with Y145. Furthermore, the V163A mutation that results in thermal stabilization of bacterially produced GFP led to a small but consistent $20 \%$ increase of the signals detected (see pairs 3 vs 28, 5 vs 18, 7 vs 1 ). The S175G mutation [10] did not result in any significant further improvement (see 19 vs 1). In several experiments, however, the signal from plasmid $\mathrm{n}^{\circ} 19$ was always equal or marginally stronger than that obtained from plasmid $n^{\circ} 1$ (not shown). The BFP form carried by plasmid ${ }^{\circ} 19$, called SBFP, was therefore chosen for further experiments.

SGFP, a green EGFP derivative carrying mutations V163A and S175G was also generated. Whilst no remarkable improvement of the signal was found (not shown), SGFP was consistently equivalent or slightly brighter than EGFP, as was observed for the $n^{\circ} 1$ and $n^{\circ} 19$ BFP versions. SGFP was therefore chosen for the next set of experiments.

A further attempt to improve the levels of fluorescence detected was made by targeting SBFP and SGFP to various cellular compartments. Targeting of EGFP to the nucleus by tagging it with the SV40 nuclear localization signal provided by pCATCH-NLS (see [14] and Exp. Proc.) led to a 30-50\% increase of the fluorescence detected by FACS (not shown). The SV40- 
tagged FPs concentrated in the nucleus, but also appeared throughout the cytoplasm (Fig. 1). This is probably because their small size (30kDa) allows some diffusion into and out of the nucleus through the nuclear pores [22].

Targeting GFP to the ER was known to improve its expression in plants [23]. SBFP and SGFP were therefore targeted to this compartment by addition of an immunoglobulin leader peptide to the N-terminus and an ER retention signal, KDEL, to the C-terminus (see Exp. Proc. for details). Transfection of ER-SBFP and ER-SGFP in COS 7 cells resulted in fluorescent staining patterns typical of ER distribution ( Fig. 2), and the levels of fluorescence detected by FACS were comparable to those obtained with the nuclear forms (Table 1). These proteins could prove very useful tools to study the dynamics of the ER compartment in live cells using approaches similar to those recently reported for the Golgi complex [24].

A final attempt to improve fluorescence by targeting was made by expressing SBFP and SGFP at the cell surface. For this, the KDEL tags were replaced by the transmembrane and cytoplasmic domain of a rat MHC class I molecule, RT1-A ${ }^{u}$ [15] (see Exp. Proc.). This led to typical cell-surface staining, with an enhanced vesicular signal in the perinuclear region, which presumably corresponds to the secretory pathway (see Fig. 1). For both SBFP and SGFP, the levels of fluorescence detected were, however, 5-10 fold lower than the corresponding nuclear and ER forms (see Table 1 and Fig. 1).

The spectral characteristics of COS 7 cells transfected with all the BFP and GFP constructs reported in this paper were analysed and, apart from $\mathrm{n}^{\circ} 3$ and 28 which were too weak to be detected, found to have spectral behaviours comparable to bacterially-produced BFP or S65T GFP [1], with an intensity proportional to the signals recorded by FACS analysis (not shown).

When different forms of SBFP and SGFP were co-transfected, they were clearly distinguished by fluorescence microscopy (Fig. 2). When nuclear SBFP was expressed together with ER-SGFP, however, the blue nuclear 
staining appeared somewhat weaker amidst the green fluorescence leaking through the 420nm long-pass filter in the standard DAPI block used (see Exp. Proc.). GFP and BFP signals are virtually non-overlapping, which is crucial if they are to be used in parallel as reporter genes. There is a significant overlap, however, between the emission spectrum of BFP and the absorption spectrum of GFP.

To test whether co-expression of BFP and GFP in a single cell could lead to a significant reabsorption of BFP's fluorescence by GFP, the various forms described above were transfected with one another in all nine possible combinations and analysed by FACS (Fig. 3). As can be seen by comparing the first row with the first column, signals from GFP and BFP are clearly distinct, and only at the highest level of green fluorescence was there a small cross-signal detected in the blue channel. In all cases where SBFP and SGFP were co-transfected, a striking correlation of the levels of blue and green fluorescence was observed, like that reported for co-transfection with lipofectin [25] or electroporation [4]. Thus, co-transfection by the DEAEdextran transfection method also leads to very efficient co-expression. Compared to single transfections, an overall $10-20 \%$ decrease of the blue and green signals was observed in the co-transfections, and appeared most marked when both forms were targeted to the ER. 


\section{Discussion:}

GFP is naturally expressed in a jellyfish at the temperature of sea water, which is usually much cooler than $37^{\circ} \mathrm{C}$. Native GFP is therefore not well suited for expression at $37^{\circ} \mathrm{C}$, and numerous mutations have been reported by various groups that all increase the thermal stability of GFP [9-12]. In this study, three such mutations (F64L, V163A and S175G) were combined. F64L[11] , which was already present in the EGFP version used as starting material, has little or no effect on the spectral properties of GFP, but seems almost indispensable for its efficient expression at $37^{\circ} \mathrm{C}[26]$. Introduction of V163A [10] led to a $20 \%$ increase of the fluorescence detected, whereas the further intoduction of S175G provided no further significant improvement. This was somewhat disappointing since, in bacteria, the conjunction of V163A and S175G had been reported to result in a fivefold improvement of fluorescence over natural GFP [9]. If the F64L mutation already resulted in a high proportion of appropriate folding at $37^{\circ} \mathrm{C}$, this would leave little room for improvement by further addition of "stabilizing" mutations, which might be the reason behind the small improvements found with V163A and S175G. For this reason, I decided to disregard several other mutations that have also been reported as potentially beneficial for the folding and stability of natural GFP at $37^{\circ} \mathrm{C}$, like F100S and/or M154T [9] or S147P [12].

Two forms of BFP are commercially available for expression in mammalian cells. Quantum Biotechnology offers a plasmid for expression of $\mathrm{BFP}$ that is not enriched for mammalian codons, and the expected protein sequence corresponds to that of plasmid $\mathrm{n}^{\circ} 10$. Clontech recently started distributing an 'EBFP' version that encodes a BFP protein whose sequence corresponds to the combination found in plasmid $n^{\circ} 5$ [27], which, at least in the system used here, is five-fold less intense than SBFP. Miyawaki et al. have also recently reported another EBFP form that is derived from a 
'humanized' GFP sequence [6], and carries a combination of substitutions corresponding to plasmid $\mathrm{n}^{\circ} 7$. The expression of EBFP on its own, however, was not documented in the paper. From the results presented in Table 1, it is therefore apparent that SBFP is a significant improvement over these three available BFP versions aimed at expression in mammalian cells.

Furthermore, if GFP or BFP are to be used as reporter genes, a further 50\% improvement of the signals can be obtained by using the nuclear- or ERtargeted forms.

In cases where direct purification of live cells expressing the reporter genes are required, expressing FPs at the cell surface would be highly desirable, but the results obtained after targetting SBFP or SGFP to the cell surface suggest that this localization is highly detrimental to the intensity of fluorescence attained.

If BFP is to be used in conjunction with GFP in a dual-colour reporter gene system, it is of primary importance to ensure that expression of one form does not markedly influence detection of the other. It the case of the GFP/BFP pair, one concern was that blue light emitted by BFP might be efficiently reabsorbed by GFP. To estimate to what extent the expression of GFP might obscure the BFP signals, the various FP versions targeted to different cellular compartments were used. The reasoning was that, if GFP reabsorbed a significant amount of the light emitted by BFP, this would be favoured by proximity, and therefore most noticeable when both forms were expressed in the same cellular compartment. The results obtained show clearly that this does not happen. When two plasmids where co-transfected, there was indeed a slight decrease in the fluorescence signals detected. Since this decrease affected green and blue signals to the same extent, however, it cannot have been due to absorption of the blue fluorescence by GFP, but was more likely due to competition of the plasmids for episomal replication in COS 7 cells. The more marked decrease observed for both blue and green 
fluorescence when ER-SBFP and ER-SGFP were transfected together suggests that, at the very high levels attained in the expression system used here, the number of KDEL receptors in the ER might be a limiting factor. From these results, one can conclude that GFP does not significantly reabsorb the blue signal emitted by BFP when they are expressed in the same cell or even in the same cellular compartment, and these pair of fluorescent proteins are therefore highly suitable as a dual-colour reporter gene system.

Acknowledgements: I am grateful to Rosario Rizzuto, Christopher Hovens and Sean Munro for providing plasmids, to Nigel Miller for expert assistance with FACS analysis, to Peter Lipp for help with printing digital images, to Kirby Siemering and Jim Haseloff for helpful discussions, and to Carol Featherstone for careful reading of the manuscript. This work was supported by a BBSRC post-doctoral fellowship.

\section{References:}

1. Heim, R., D.C. Prasher, and R.Y. Tsien, Wavelength mutations and posttranslational autoxidation of green fluorescent protein. Proceedings Of the National Academy Of Sciences Of the United States Of America, 1994. 91(26): p. 12501-12504.

2. Heim, R. and R.Y. Tsien, Engineering green fluorescent protein for improved brightness, longer wavelengths and fluorescence resonance energy-transfer. Current Biology, 1996. 6(2): p. 178-182.

3. Rizzuto, R., et al., Double-labeling of subcellular structures with organelle-targeted GFP mutants in-vivo. Current Biology, 1996. 6(2): p. 183-188.

4. Lybarger, L., et al., Dual-color flow cytometric detection of fluorescent proteins using single-laser (488-nm) excitation. Cytometry, 1998. 31(3): p. 147-152.

5. Haas, J., E.C. Park, and B. Seed, Codon usage limitation in the expression of HIV-1 envelope glycoprotein. Current Biology, 1996. 6(3): p. 315-324. 
6. Zolotukhin, S., et al., A humanized green fluorescent protein cdna adapted for high-level expression in mammalian-cells. Journal Of Virology, 1996. 70(7): p. 4646-4654.

7. Yang, T.T., L.Z. Cheng, and S.R. Kain, Optimized codon usage and chromophore mutations provide enhanced sensitivity with the green fluorescent protein. Nucleic Acids Research, 1996. 24(22): p. 45924593.

8. Zhang, G.H., V. Gurtu, and S.R. Kain, An enhanced green fluorescent protein allows sensitive detection of gene-transfer in mammalian-cells. Biochemical and Biophysical Research Communications, 1996. 227(3): p. 707-711.

9. Crameri, A., et al., Improved green fluorescent protein by molecular evolution using DNA shuffling. Nature Biotechnology, 1996. 14(3): p. 315-319.

10. Siemering, K.R., et al., Mutations that suppress the thermosensitivity of green fluorescent protein. Current Biology, 1996. 6(12): p. 16531663.

11. Cormack, B.P., R.H. Valdivia, and S. Falkow, FACS-optimized mutants of the green fluorescent protein ( $g f p)$. Gene, 1996. 173(1): p. 33-38.

12. Kimata, Y., et al., A novel mutation which enhances the fluorescence of green fluorescent protein at high temperatures. Biochemical and Biophysical Research Communications, 1997. 232(1): p. 69-73.

13. Heim, R., A.B. Cubitt, and R.Y. Tsien, Improved green fluorescence. Nature, 1995. 373(6516): p. 663-664.

14. Georgiev, O., et al., Two versatile eukaryotic expression vectors permitting epitope tagging, radiolabeling and nuclear-localization of expressed proteins. Gene, 1996. 168(2): p. 165-167.

15. Joly, E., et al., Isolation of a functional cDNA encoding the RTI.A MHC Class I heavy chain by a novel PCR-based method. Immunogenetics, 1995. 41(5): p. 326-328.

16. Kirsch, R.D. and E. Joly, An improved PCR-mutagenesis strategy for two-site mutagenesis or sequence swapping between related genes. Nucleic Acids Research, 1998. 26(7): p. 1848-1850.

17. Gonzalez, A.L. and E. Joly, A simple procedure to increase efficiency of DEAE-dextran transfection of COS cells. Trends In Genetics, 1995. 11(6): p. 216-217.

18. Bierhuizen, M.F.A., et al., Green fluorescent protein variants as markers of retroviral-mediated gene transfer in primary hematopoietic cells and cell lines. Biochemical and Biophysical Research Communications, 1997. 234(2): p. 371-375. 
19. Kozak, M., At least 6 nucleotides preceding the AUG initiator codon enhance translation in mammalian cells. Journal Of Molecular Biology, 1987. 196(4): p. 947-950.

20. Miyawaki, A., et al., Fluorescent indicators for Ca2+ based on green fluorescent proteins and calmodulin. Nature, 1997. 388(6645): p. 882887.

21. Aruffo, A. and B. Seed, Molecular-cloning of a CD28 cDNA by a high-efficiency COS cell expression system. Proceedings Of the National Academy Of Sciences Of the United States Of America, 1987. 84(23): p. 8573-8577.

22. Chatterjee, S. and U. Stochaj, Monitoring nuclear transport in Helacells using the green fluorescent protein. Biotechniques, 1996. 21(1): p. 62-63.

23. Haseloff, J., et al., Removal of a cryptic intron and subcellular localization of green fluorescent protein are required to mark transgenic Arabidopsis plants brightly. Proceedings Of the National Academy Of Sciences Of the United States Of America, 1997. 94(6): p. 2122-2127.

24. Cole, N.B., et al., Diffusional mobility of Golgi proteins in membranes of living cells. Science, 1996. 273(5276): p. 797-801.

25. Ropp, J.D., et al., Aequorea green fluorescent protein-simultaneous analysis of wild-type and blue-fluorescing mutant by flow-cytometry. Cytometry, 1996. 24(3): p. 284-288.

26. Palm, G.J., et al., The structural basis for spectral variations in green fluorescent protein. Nature Structural Biology, 1997. 4(5): p. 361-365.

27. Yang, T.T., et al., Improved fluorescence and dual color detection with enhanced blue and green variants of the green fluorescent protein. Journal of Biological Chemistry, 1998. 273(14): p. 8212-8216. 


\section{Legends:}

Table 1: FACS analysis of the various modified BFP forms. Transiently transfected COS 7 cells were fixed with $1 \%$ formaldehyde 48 hour after transfection, and analysed on a FACS Vantage flow cytometer. The means of fluorescence indicated in column 8 are calculated from the cells staining positively, which amounted to $40-60 \%$ of the whole population in this experiment. For the plasmids on the first four lines, the weak blue fluorescence overlapped largely with the autofluorescence spectrum of untransfected cells, and means therefore had to be estimated. The index of specific fluorescence is obtained by the following formula: (mean fluorescence of positive cells - mean fluorescence of untransfected control) / mean fluorescence of untransfected control. Comparable results were obtained in more than 3 other experiments.

Fig. 1: COS 7 cells transiently transfected with plasmids for the expression of various forms of SBFP or SGFP.

All the pictures shown were taken on unfixed, living cells. The first two panels show successive $10 \mathrm{sec}$. exposures of SBFP targeted to the nucleus, in order to demonstrate that only moderate bleaching occurs.

The third panel shows SBFP targeted to the ER (20 sec. exp).

On the second line, the first picture shows SGFP targeted to the nucleus (5 sec. exp). In the cell undergoing mitosis on the right side of that picture, disassembly of the nucleus results in a bright signal distributed throughout the cytoplasm. The second panel shows SGFP targeted to the ER (5 sec. exp.). The very fine ER structure is best captured on live cells. The shorter exposure required with ER-SGFP compared to ER-SBFP also contributes to the finer resolution obtained. The third picture shows SGFP targeted to the cell surface, attached to the transmembrane domain of the rat MHC class I molecule RT1-Au (30 sec. exp.). 
Fig. 2: Co-transfection of SBFP and SGFP targeted to different cellular compartments.

Line 1: Co-transfection of ER-SBFP (right panel) and nls-SGFP (left panel)

Line 2: Co-transfection of ER-SGFP (right panel) and nls-SBFP (left panel). In this last picture, the very bright green ER signal 'leaks' through the standard DAPI filter set used (see Exp. Proc.), and somewhat obscures perception of the blue nucleus.

Fig. 3: FACS analysis of COS 7 cells co-transfected with SGFP and SBFP targeted to various cellular compartments.

Blue fluorescence is measured on the $\mathrm{x}$ axis (FL4). Green fluorescence is measured on the y axis (FL1).

nls-: targeted to the nucleus by an SV40 nuclear localization signal.

ER-: retained in the ER by addition of a C-terminal KDEL sequence.

$\mathrm{Au}-$ : expressed at the cell surface attached to the transmembrane and intracellular portion of the rat MHC class I molecule RT1-A ${ }^{\mathrm{u}}$.

Each panel shows $10^{4}$ cells gated on FSC/SSC. Cells were fixed in PBS/1\% formaldehyde before analysis. 
Table 1

\begin{tabular}{|c|c|c|c|c|c|c|c|c|}
\hline $\begin{array}{c}\text { plasmid } \\
\text { number }\end{array}$ & $\begin{array}{c}\text { name } \\
\text { if any) }\end{array}$ & $\begin{array}{c}\text { Cellular } \\
\text { localisation }\end{array}$ & $64-65-66$ & 145 & 163 & 175 & $\begin{array}{c}\text { mean } \\
\text { fluorescence }\end{array}$ & $\begin{array}{c}\text { index of } \\
\text { specific } \\
\text { fluorescence }\end{array}$ \\
\hline 3 & & nls & LTH & Y & V & S & $c a .10$ & 1 \\
\hline 28 & & nls & LTH & Y & A & S & $c a .15$ & 2 \\
\hline 5 & & nls & LTH & F & V & S & $c a .25$ & 4 \\
\hline 18 & & nls & LTH & F & A & S & $c a .30$ & 5 \\
\hline 10 & & nls & LSH & Y & A & S & 72 & 13 \\
\hline 7 & & nls & LSH & F & V & S & 83 & 16 \\
\hline 1 & & nls & LSH & F & A & S & 109 & 20 \\
\hline 19 & SBFP & nls & LSH & F & A & G & 110 & 20 \\
\hline 2 & ER-SBFP & ER & LSH & F & A & G & 110 & 20 \\
\hline 4 & A u-SBFP & CS & LSH & F & A & G & $c a .30$ & 5 \\
\hline
\end{tabular}




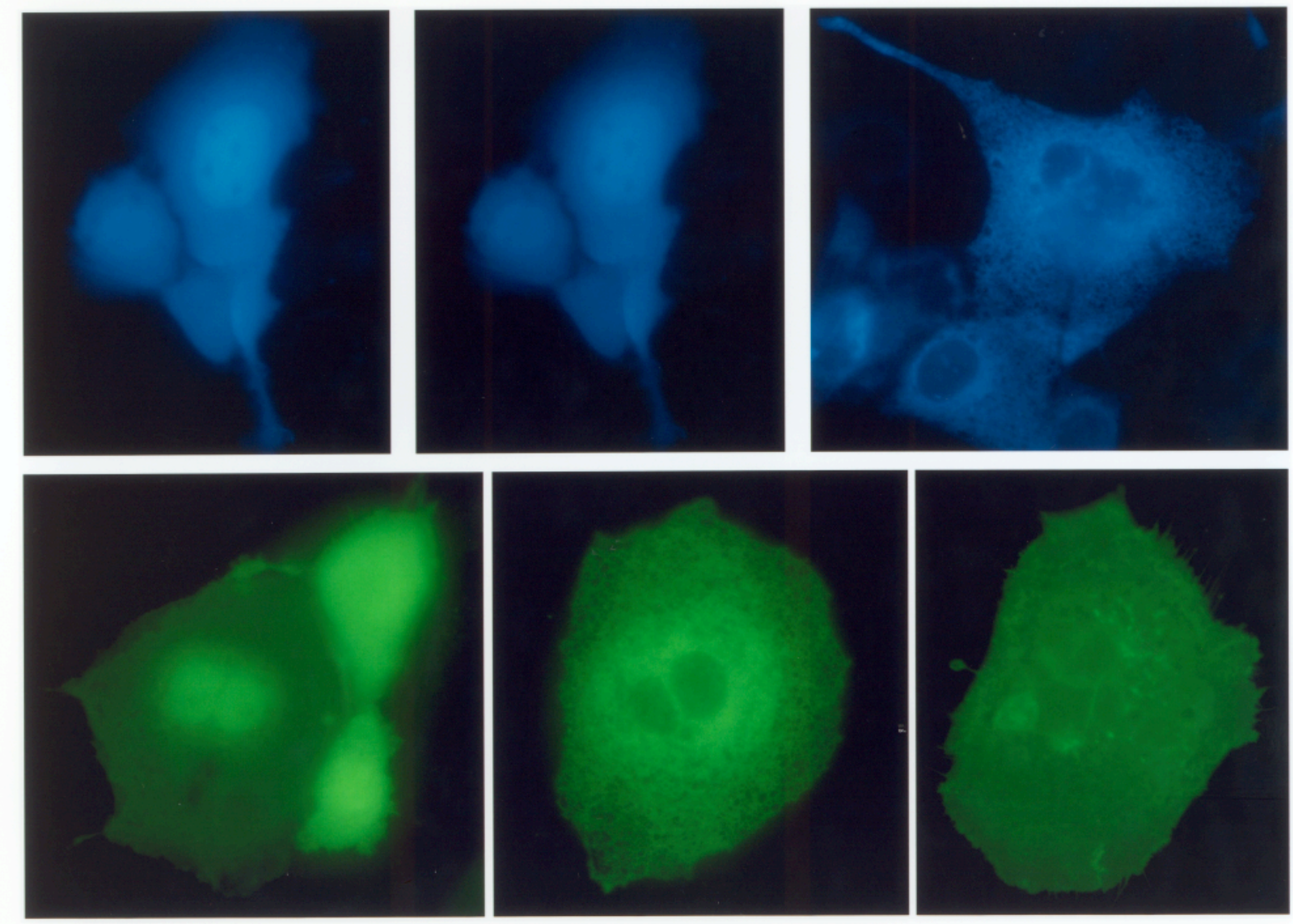




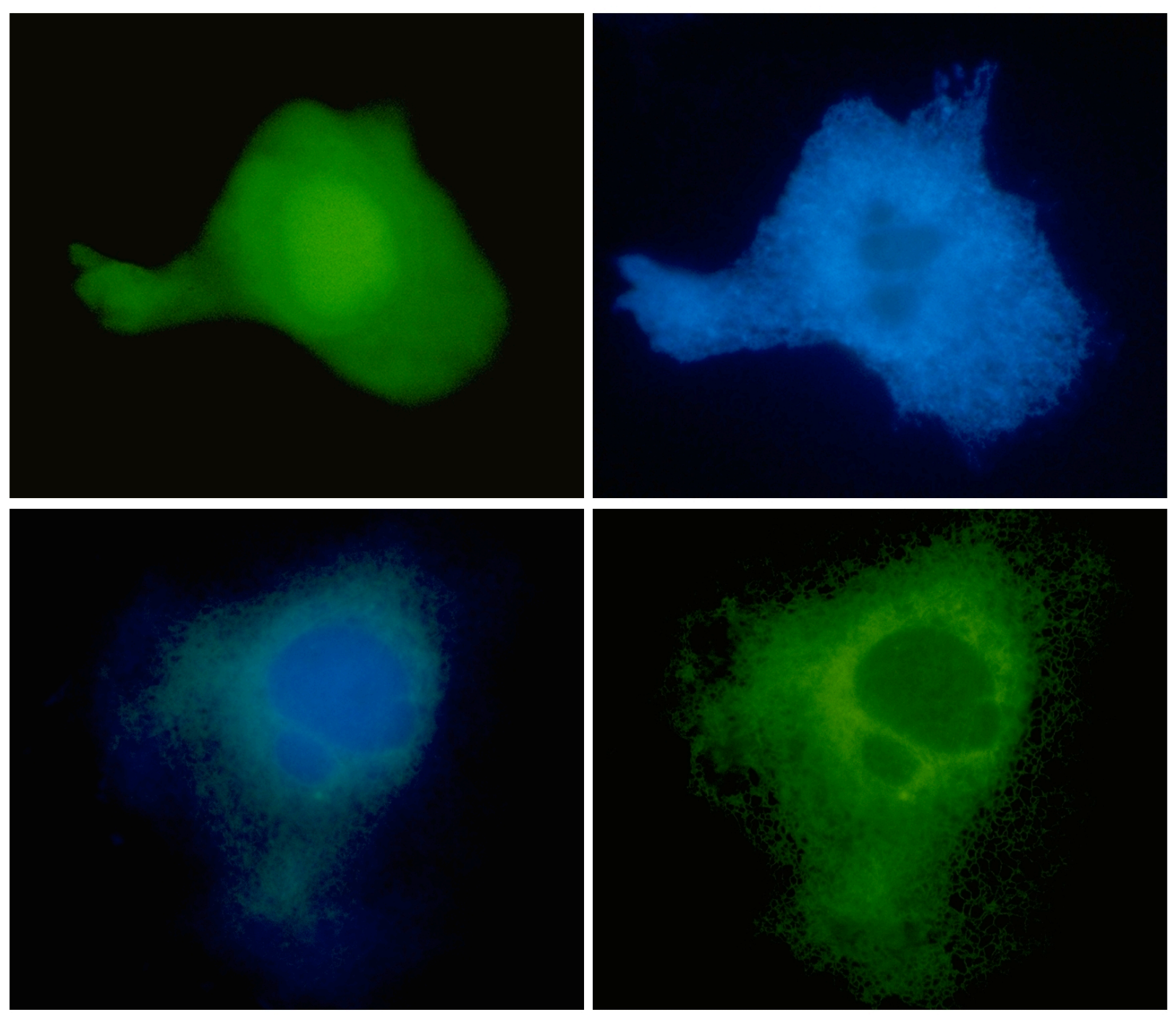



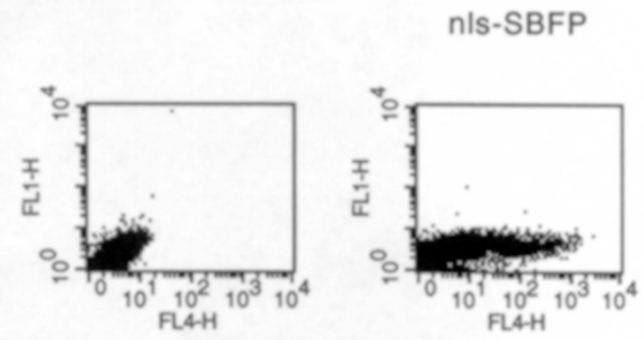

ER-SBFP
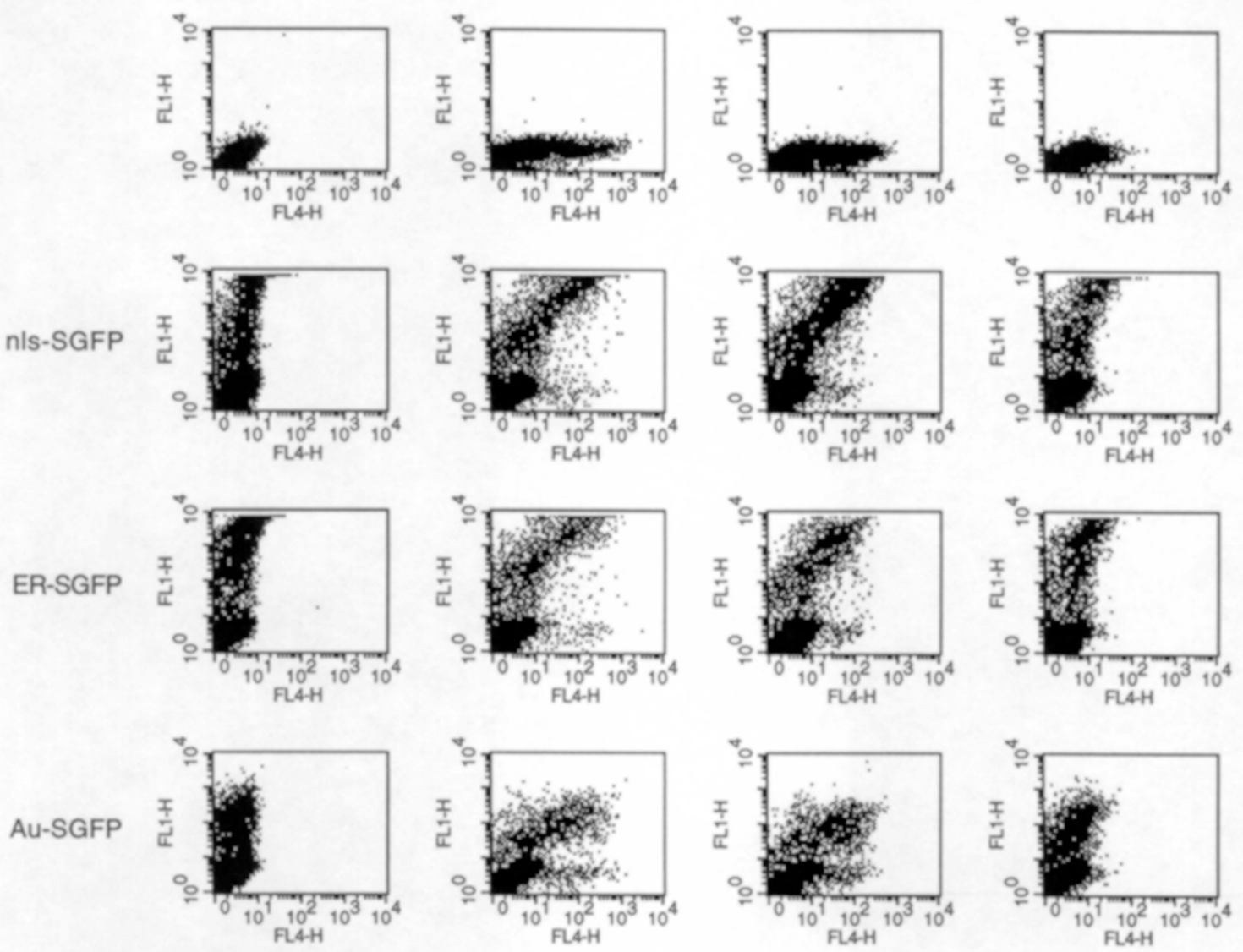


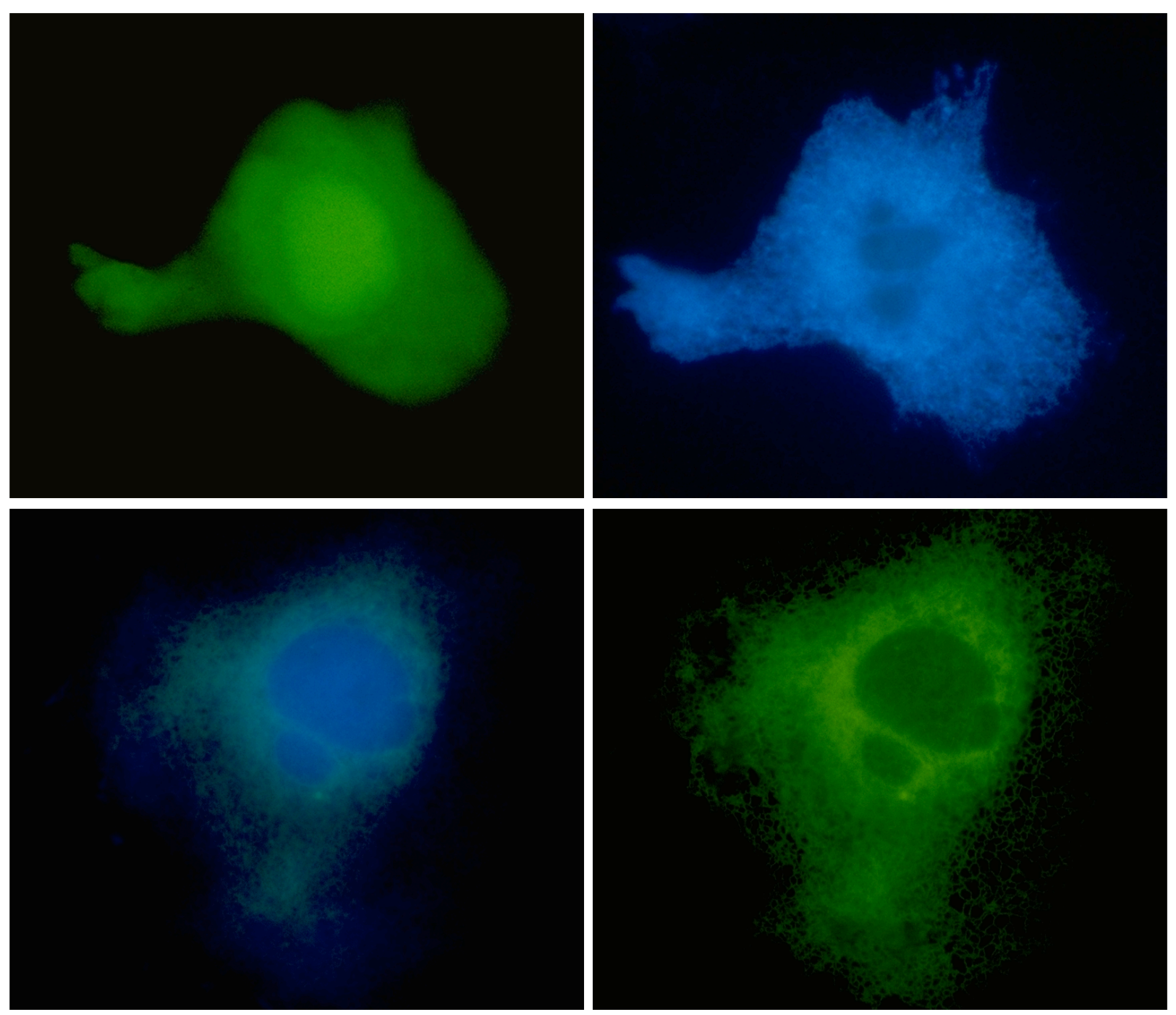

